PUJADAS SALVÀ, A.J. -2006- Nuevas combinaciones nomenclaturales y aportaciones florísticas en el género Orobanche L. (Orobanchaceae). Lagascalia 26: 246-248.

ROMÁN B, C. ALFARO, A. TORRES, Z. SATOVIC, A. PUJADAS \& D. RUBIALES 2003- Genetic relationship among Orobanche species as revealed by RAPD analysis. Ann. Bot. 91: 637-642.

SCHNEEWEISS, G.M., A.E. COLWELL, J.M. PARK, C. JANG \& T. STUESSY -2004aPhylogeny of holoparasitic Orobanche (Orobanchaceae) inferred from nuclear ITSsequences. Mol. Phylogenet. Evol. 30: 465478.

SCHNEEWEISS, G.M, T. PALOMEQUE, A.E. COLWELL \& H. WEISS-SCHNEEWEISS 2004b- Chromosome numbers and karyotype evolution in holoparasitic Orobanche (Orobanchaceae) and related genera. Am. J. Bot. 91: 439-448.

SCHNEEWEISS, G. M., J. M. PARK, J. F.
MANEN, A. E. COLWELL \& H. WEISSSCHNEEWEISS -2005- Phylogenetic relationships of Orobanche and related genera: evidence from molecular and karyological data., in Burckhardt \& Mühlethaler (eds.), 8th GfBS Annual Conference Abstracts. Org. Divers. Evol. 5, Electr. Suppl. 13: 88.

TZVELEV, N. -1981- Orobanchaceae, in Fedorov (ed.), Fl. Evropeiskoi- Chasti SSSR, 5: 317-336. Leningrad.

WEISS-SCHNEEWEISS, H., J. GREILHUBER \& G. M. SCHNEEWEISS -2006- Genome size evolution in holoparasitic Orobanche (Orobanchaceae) and related genera. Am. J. Bot. 93: 148-156.

Dirección del autor. Departamento de Ciencias y Recursos Agrícolas y Forestales. Edificio Celestino Mutis. Campus de Rabanales. Universidad de Córdoba. E-14071 Córdoba. Email: cr1pusaa@uco.es

\title{
130. ORCHIS CAZORLENSIS LACAITA EN EL PARQUE NATURAL DE LA SIERRA DE LAS NIEVES (MÁLAGA)
}

Francisco Javier BORREGO AGUAYO, Antonio LÓPEZ PASTORA, Manuel MELGAR RUEDA, Sergio PRATS FONS, Gerardo SIERRA DE CÓZAR y Juan TERROBA VALADÉS

Recibido el 30 de abril de 2007, aceptado para su publicación el 4 de junio de 2007

Publicado "on line" en junio de 2007

Orchis cazorlensis Lacaita in the Natural Park Sierra de las Nieves (Málaga)

Palabras clave. Orchidaceae, Orchis cazorlensis, Serranía de Ronda, Sierra de las Nieves, Málaga.

Key words. Orchidaceae, Orchis cazorlensis, Serranía de Ronda, Sierra de las Nieves, Málaga.

Orchis cazorlensis Lacaita (fig. 1) es un taxon endémico de la Península Ibérica que en Andalucía se ha citado para las provincias de Jaén y Granada, siendo dudosa su presencia en Almería. Ésta sería la primera cita de la especie para la provincia de Málaga 


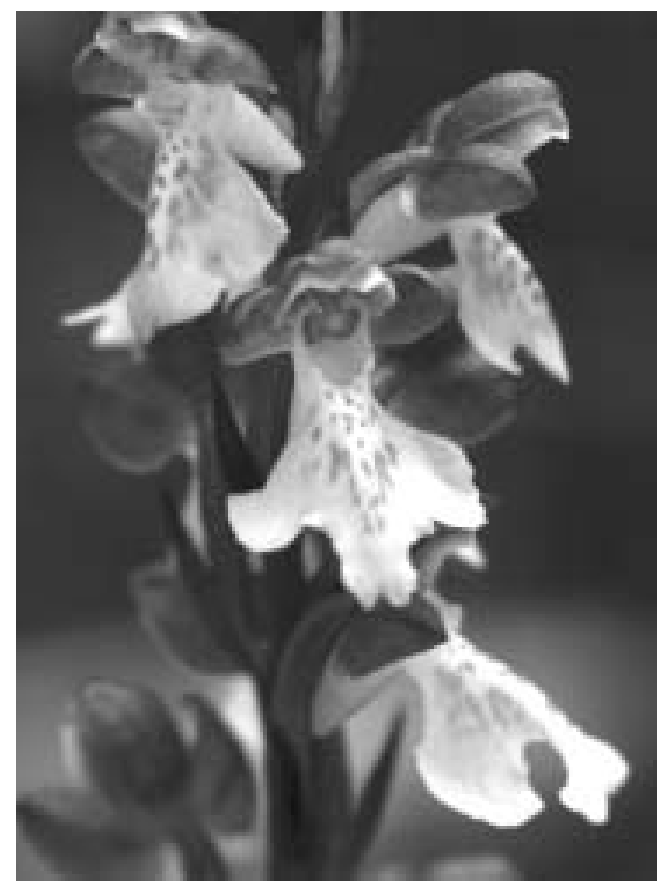

Figura 1. Orchis cazorlensis Lacaita. Detalla de las flores.Orchis cazorlensis Lacaita. Detail of the flowers.

(Aedo, 2005; Lowe, 1998)

ESPAÑA: MÁlAGA. Parauta. P. N. Sierra de Las Nieves; Carril de Tolox, cuenca superior del río Verde, entre 1195 y 1220 m. Vista por primera vez en abril de 2006, y recolectada el 10 de mayo de 2007. F. Borrego, A. López, M. Melgar, S. Prats, G. Sierra y J. Terroba. 30SUF1795060500. (MGC 65862 y 65863).

La población localizada en 2006 se componía de 16 ejemplares, extendida en un radio de $100 \mathrm{~m}$ alrededor del punto mencionado, en laderas de no mucha inclinación (5-20\%), orientadas al N o NE y sobre rocas calcáreo-dolomíticas (Brecha de La Nava). Hemos observado su floración de abril a mayo.
La especie se desarrolla en el seno de una comunidad, sobre dolomías, de pinos carrascos, sabinas y pinsapos en la que predominan los matorrales de aulagas y espliegos. Las especies más importantes de esta comunidad (Abieto pinsapoJuniperetum phoeniceae pinetosum halepensis Pérez Latorre y Cabezudo 1999) son:

Pinus halepensis, Abies pinsapo, Juniperus phoenicea, Juniperus oxycedrus, Quercus rotundifolia, Paeonia broteri, Cerastium gibraltaricum, Cistus albidus, Ulex baeticus, Rhamnus myrtifolius, Bupleurum spinosum, Hedera helix, Phyllirea angustifolia, Daphne gnidium, Putoria calabrica, Thymus mastichina, Phlomis crinita var malacitana, Lavandula lanata, Linaria tristis, Staehelina dubia, Santolina canescens, Ptilostemon hispanicum, Leuzea conifera, Klasea pinnatifida, Senecio minutus, Stipa tenacissima, Festuca plicata, Ruscus aculeatus, Hyacinthoides hispanica, Gagea foliosa subsp. elliptica, Aceras anthropophorum, Ophrys fusca subsp fusca, Ophrys scolopax, Neotinea maculata, Orchis mascula, Orchis langei, Orchis conica y Orchis italica.

\section{BIBLIOGRAFÍA}

AEDO, C. -2005- Orchis L., in Aedo, C. \& Herrero, A. (eds.)., Flora Ibérica, 21: 136. Madrid.

LOWE, M. R. -1998- The orchids of the province of Málaga, Spain. J. Eur. Orch. 30(3): 501570.

Dirección de los autores. Apdo Correos 1, 29400 Ronda. Tel. 952874426

Autor para correspondencia: mandraagora@gmail.com 